
p. 541-543, dezembro 2002 .

\title{
Resistência a abamectin e cartap em populações de traça-das-crucíferas
}

\author{
Marina Castelo Branco; Cynthia A. de Melo \\ Embrapa Hortaliças, C. Postal 218, 70.359-970 Brasília-DF
}

\section{RESUMO}

A suscetibilidade de populações de traça-das-crucíferas a cartap e abamectin provenientes de cinco campos de repolho, com diferentes histórias de uso de inseticidas, foi comparada através de um bioensaio em que se usou a imersão das folhas em caldas de inseticidas. Foram utilizadas seis concentrações para cada produto, mais um tratamento água como controle, com três a sete repetições. Discos de folha de repolho ( $2 \mathrm{~cm}$ de diâmetro) retirados de plantas com 35 dias de idade foram imersos nas caldas por 10 segundos. Após secarem por duas horas, os discos foram colocados em placas de Petri e 10 larvas de segundo estádio de traça-das-crucíferas foram colocadas sobre estes. Após isto, as placas foram mantidas em uma câmara com temperatura de $20 \pm 1^{\circ} \mathrm{C}$ e fotofase de $13 \mathrm{~h}$. A mortalidade larval foi avaliada após $48 \mathrm{~h}$ para cartap e $72 \mathrm{~h}$ para abamectin. Os resultados dos ensaios foram avaliados usando o método "probit". Os índices de resistência foram calculados dividindo-se a $\mathrm{CL}_{50}$ de cada população pela $\mathrm{CL}_{50}$ da população mais suscetível. A população mais suscetível foi coletada em área sem aplicação destes produtos. $O$ índice de resistência para cartap variou de 2,8 a 7,1, sendo que este último foi observado em uma população coletada em área tratada diversas vezes com o inseticida. Os índices de resistência para abamectin variou de 1,1 a 12,1 ; porém, a população mais resistente foi coletada em área sem nenhuma aplicação do produto. Uma das causas deste resultado poderia ser a migração de indivíduos resistentes para a área de cultivo.

Palavras-chave: Brassica oleracea var. capitata, repolho, Plutella xylostella, resistência a inseticida.

\author{
ABSTRACT \\ Susceptibility to cartap and abamectin in Diamondback \\ Moth strains
}

Susceptibility of Diamondback Moth to cartap and abamectin, from five areas with different histories of insecticide spray, was compared using a leaf-dip bioassay. Six concentrations of each insecticide plus water control were evaluated with three to seven replications per concentration. Cabbage leaf disks ( $2 \mathrm{~cm}$ diameter) from 35 -day old plants were immersed in test solutions for $10 \mathrm{~s}$. The disks were allowed to air dry for $2 \mathrm{~h}$ and then placed in Petri dishes. Ten second-instar larvae were then transferred to each disk. After the treatments insects were maintained in a chamber at temperatures of $20 \pm 1^{\circ} \mathrm{C}$ and a photoperiod of 13L:11D. Larval mortality was assessed after $48 \mathrm{~h}$ of cartap treatment and after $72 \mathrm{~h}$ of abamectin treatment. Bioassay results for each insecticide were analysed using the probit method. Resistance ratios were calculated dividing the $\mathrm{LC}_{50}$ of each strain by the $\mathrm{LC}_{50}$ of the most susceptible one, which was collected in a field with no insecticide spray. The resistance ratio to cartap ranged from 2.8 to 7.1 , with the most resistant strain coming from a field sprayed several times with the insecticide. To abamectin, the resistance ratios ranged from 1.1 to 12.1 . The most resistant strain was from a field without abamectin spray. One cause of this result could be the migration of resistant insects into the field.

Keywords: Brassica oleracea var. capitata, cabbage, Plutella xylostella, Diamondback Moth, insecticide resistance.

(Recebido para publicação em 20 de junho de 2000 e aceito em 12 de setembro de 2002)

A traça-das-crucíferas (TDC), devido às perfurações que causa nas cabeças de repolho, é considerada a principal praga da cultura. Para reduzir os danos desta praga, diversos inseticidas são utilizados. Abamectin foi testado e apresentou resultado satisfatório em uma ocasião (Castelo Branco \& Guimarães, 1990) e insatisfatório em outra (França \& Medeiros, 1998). Neste caso, a ineficiência do produto foi atribuída à elevada densidade populacional da praga por ocasião da realização do ensaio. Para cartap, o mesmo tipo de comportamento foi observado: eficiência em um ensaio (Castelo Branco, 1997) e ineficiência em outro (Castelo Branco \& Guimarães, 1990).

Ainda que os resultados de eficiência destes inseticidas tenham sido variáveis, em alguns locais do Brasil estes produtos são utilizados para o controle da TDC em pulverizações intensivas. O elevado número de pulverizações pode levar à seleção de populações resistentes, como já foi observado para abamectin na China (Zhang et al., 2001) e na Malásia (Iqbal et al., 1996) e para cartap na Malásia (Sudderuddin \& Kok, 1978), em Taiwan (Cheng, 1981) e no Japão (Hama et al., 1992). A seleção de populações resistentes pode inviabilizar o uso dos produtos.

Afora isto, o conhecimento do grau de resistência de populações de TDC aos inseticidas empregados para o seu controle é importante para acompanhar a evolução da resistência ao longo do tempo, bem como para a implementação de programas de manejo de resistência. Por isso, este trabalho teve como objetivo avaliar o nível de suscetibilidade de cin- co populações de TDC a cartap e abamectin, sendo três populações do Distrito Federal, uma de Barroso (MG) e outra de Tianguá (CE).

\section{MATERIAL E MÉTODOS}

Larvas e pupas de TDC foram coletadas em campos de repolho entre setembro e novembro de 1999. Três populações do inseto foram coletadas no Distrito Federal (populações Embrapa Hortaliças, Taquara e Brazlândia), uma no município de Barroso (população Barroso) e outra no município de Tianguá (população Tianguá). Os agricultores de cada área foram entrevistados para a obtenção de informações sobre os inseticidas utilizados e a freqüência de aplicação nos quatro meses que antecederam a coleta das populações. 
Tabela 1: Suscetibilidade de cinco populações de Plutella xylostella a cartap. Brasília, Embrapa Hortaliças, 1999.

\begin{tabular}{lcccc}
\hline \multicolumn{1}{c}{ População } & $\mathbf{n}^{1}$ & $\mathbf{C L 5 0}^{\mathbf{2}} \mathbf{( I C 9 5 \%}^{\mathbf{3}}$ (g.i.a./ha) & Inclinação $\pm \mathbf{D P}^{4}$ & FR $^{\mathbf{5}}$ \\
\hline Embrapa Hortaliças & 127 & $18,0(10,5-25,5)$ & $2,4 \pm 0,5$ & 1 \\
Barroso & 257 & $50,0(41,0-58,5)$ & $4,2 \pm 0,6$ & 2,8 \\
Taquara & 240 & $56,5(39,0-80,5)$ & $3,0 \pm 0,3$ & 3,2 \\
Tianguá & 231 & $70,0\left(\right.$ nd $\left.^{6}\right)$ & $1,7 \pm 0,2$ & 3,8 \\
Brazlândia & 242 & $128,0(98,5-160,0)$ & $2,1 \pm 0,3$ & 7,1 \\
\hline
\end{tabular}

${ }_{1}^{1}$ Número total de insetos testados excetuando os do tratamento testemunha.

${ }^{2 /}$ Concentração letal para $50 \%$ da população

${ }^{3}$ Intervalo de confiança $95 \%$

${ }^{4 /}$ Desvio padrão

${ }^{5 /}$ Fator de resistência (CL50 população/ CL50 população mais suscetível)

${ }^{6 /} \mathrm{nd}=$ não determinado.

As larvas e pupas obtidas no campo foram criadas no laboratório de Entomologia da Embrapa Hortaliças sobre folhas de repolho até a obtenção da primeira geração, a qual foi utilizada nos bioensaios de laboratório. Para a determinação da concentração-resposta das populações aos inseticidas foram preparadas soluções onde as concentrações utilizadas foram: a) cartap: 600; $300 ; 150 ; 75 ; 37,5$ e 3,75 g.i.a./ha; b) abamectin: 10,$8 ; 5,4 ; 0,54 ; 0,27 ; 0,027$ a 0,0027 g.i.a./ha (para a população Barroso não foi empregada a dosagem de 0,0027 g. i.a./ha). Dependendo do número de larvas disponíveis para cada população, foram utilizadas três a sete repetições em um ou dois dias, sendo que a cada dia foram uitlizadas no mínimo duas repetições. Para todas as repetições foi incluído um tratamento testemunha (água + espalhante). As soluções dos inseticidas foram feitas tomando-se como padrão um volume de 4001 de calda/ha. Espalhante adesivo $(20 \mathrm{ml} /$ 1001 de água) foi adicionado a todas as soluções.

Discos de folhas de repolho de $2 \mathrm{~cm}$ de diâmetro provenientes de plantas de repolho cv. Kenzan com 35 dias de idade foram imersos nas soluções por 10 seg. e em seguida postos a secar a temperatura ambiente por cerca de duas horas. Quando secos, os discos foram transferidos individualmente para placas de Petri de $9 \mathrm{~cm}$ de diâmetro e 10 larvas de segundo estádio do inseto foram colocadas sobre cada disco.

As placas de Petri contendo as larvas foram armazenadas em uma câmara climatizada com temperatura de $20^{\circ} \mathrm{C}$ $\pm 0,5^{\circ} \mathrm{C}$ e fotofase de $13 \mathrm{~h}$ até o momento da avaliação da taxa de mortalidade larval. Para cartap, a avaliação foi realizada $48 \mathrm{~h}$ após o tratamento e após $72 \mathrm{~h}$ para abamectin, visto que resultados anteriores mostraram que este inseticida apresenta uma resposta mais lenta para as dosagens menores.

Os dados de mortalidade foram corrigidos pela fórmula de Abbott (1925) sendo que foi definido previamente que onde a mortalidade na testemunha fosse superior a $20 \%$ a repetição seria descartada. Após a correção da mortalidade os dados foram analisados pelo método de Finney (1971). Foi determinada a $\mathrm{CL}_{50}$ (dose capaz de causar a mortalidade de $50 \%$ da população) e as populações foram consideradas significativamente diferentes se o intervalo de confiança a $95 \%$ (IC95\%) da $\mathrm{CL}_{50}$ das populações não se sobrepusessem. A razão de resistência (RR) foi obtida dividindo-se a $\mathrm{CL}_{50}$ de cada população pela $\mathrm{CL}_{50}$ da população mais suscetível encontrada para cada inseticida.

\section{RESULTADOS E DISCUSSÃO}

A entrevista com agricultores sobre a freqüência de uso de inseticidas revelou que a área da Embrapa Hortaliças foi a única onde estes produtos não foram utilizados. Em Brazlândia foi observado um esquema de pulverizações semanais alternadas com cartap e fentoato; em Barroso eram realizadas duas aplicações por semana, em rotação, com os seguintes produtos: clorfluazuron, deltametrina, paration metil, fenpropatrin ou metomil; em
Taquara eram realizadas de três a sete aplicações por semana com abamectin, clorfluazuron, deltametrina ou metamidofós; em Tianguá o agricultor utilizava semanalmente uma mistura de inseticidas (teflubenzuron+ metamidofós). Ainda que metamidofós seja registrado para o controle da traçadas-crucíferas, este último produtor revelou que utilizava este produto apenas para o controle de pulgões. Relatou ainda que metamidofós havia sido eficiente para o controle da traça-das-crucíferas no passado, porém agora não era mais eficiente, já que o inseto "tinha ficado forte" ao produto. Por isso ele havia começado a utilizar teflubenzuron para o controle da TDC.

A população Embrapa Hortaliças apresentou a menor $\mathrm{CL}_{50}$ para abamectin e cartap (Tabelas 1,2) e foi significativamente diferente das demais populações avaliadas, excetuando-se a população Tianguá, para o inseticida cartap (Tabela 1), para a qual o IC95\% não foi determinado. Esta população foi considerada a mais suscetível e a ela foi atribuída a razão de resistência (RR) 1 .

Para cartap, razão de resistência variou de 2,8 a 7,1, sendo a população Brazlândia a mais resistente (Tabela 1). Como este produto era intensamente utilizado na área, isto indica um processo de seleção local. Este resultado é semelhante ao observado por Liu et al. (1981) e Tabashnik et al. (1987) em Taiwan e no Havaí, respectivamente.

Para abamectin, a razão de resistência variou de 1,1 a 12,1, sendo a população Barroso a mais resistente (Tabela 2). $O$ inseticida abamectin não era utili- 
Tabela 2. Suscetibilidade de cinco populações de Plutella xylostella a abamectin. Brasília, Embrapa Hortaliças, 1999.

\begin{tabular}{lcccc|}
\hline \multicolumn{1}{c}{ Populaçăo } & $\mathbf{n}^{1}$ & $\mathbf{C L 5 0}^{2}(\mathbf{I C 9 5} \%)^{3}$ (g.i.a./ha) & Inclinaçăo $\pm \mathbf{D P}^{4}$ & FR $^{\mathbf{5}}$ \\
\hline Embrapa Hortaliças & 233 & $0,07(0,05-0,11)$ & $1,8 \pm 0,2$ & 1 \\
Brazlåndia & 398 & $0,08\left(\mathrm{nd}^{5}\right)$ & $1,6 \pm 0,3$ & 1,1 \\
Taquara & 420 & $0,15(0,12-0,19)$ & $2,2 \pm 0,2$ & 2,1 \\
Tianguá & 310 & $0,18(0,12-0,25)$ & $1,9 \pm 0,4$ & 2,3 \\
Barroso & 114 & $0,91(0,39-1,58)$ & $1,7 \pm 0,3$ & 12,1 \\
\hline
\end{tabular}

${ }^{1 / N u ́ m e r o ~ t o t a l ~ d e ~ i n s e t o s ~ t e s t a d o s ~ e x c e t u a n d o ~ o s ~ d o ~ t r a t a m e n t o ~ t e s t e m u n h a . ~}$

${ }^{2 /}$ Concentração letal para $50 \%$ da população

${ }^{3 /}$ Intervalo de confiança $95 \%$

${ }^{4 /}$ Desvio padrão

${ }^{5 /}$ Fator de resistência (CL50 população/ CL50 população mais suscetível)

${ }^{6 / n d}=$ não determinado.

zado neste local, o que sugere que a seleção da população resistente não foi feita localmente. Este resultado é semelhante ao obtido por Shelton et al. (1993) nos EUA, onde populações de TDC tratadas com Bacillus thuringiensis apresentaram níveis de resistência semelhantes a populações não tratadas. Nestes casos, a migração de indivíduos resistentes, provenientes de áreas tratadas com o produto, pode explicar o resultado encontrado. De acordo com Caprio \& Tabashnik (1992), indivíduos resistentes podem se dispersar entre áreas de cultivo, afetando o nível de suscetibilidade de populações não tratadas.

Os resultados encontrados neste trabalho indicam que programas para o manejo de resistência a inseticidas em TDC devem ser implementados no país. Para isso, deve-se incentivar a rotação de inseticidas, com a utilização de produtos que possuam diferentes mecanismos de ação e também o uso sincronizado de inseticidas nas várias regiões de cultivo; além do mais estes produtos devem ser utilizados com intervalos de alternância de 21 dias, a fim de cobrir uma geração completa da praga (Castelo Branco \& França, 2000). Além disso, o nível de suscetibilidade das populações aos diferentes produtos empregados deve ser monitorado, a fim de que os melhores produtos para cada local sejam indicados.

\section{AGRADECIMENTOS}

Os autores agradecem ao Dr. Félix H. França pela revisão do texto. A Hozanan P. Chaves e Adiel L. dos Santos pelo auxílio nos trabalhos de campo e laboratório.

\section{LITERATURA CITADA}

ABBOTT, W.S. A method of computing the effectiveness of an insecticide. Journal of Economic Entomology, v. 18, p. 265-267, 1925.

CAPRIO, M.A.; TABASHNIK, B.E. Gene flow accelerates local adaptation among finite populations: simulating the evolution of insecticide resistance. Journal of Economic Entomology, v. 85, p. 611-620, 1992.

CASTELO BRANCO, M. Insecticide resistance in Plutella xylostella (Linnaeus) (Yponomeutidae) in the Federal District, Brazil: effects of local and long-range movements of moths. Bangor. University of Wales, 1997, $196 \mathrm{p}$. (Tese doutorado)

CASTELO BRANCO, M.; FRANÇA, F.H. Previsão da eficiência de inseticidas para o controle da traça-das-crucíferas através do uso de doses discriminantes, Embrapa Hortaliças, Brasília (Boletim de Pesquisa 2 da Embrapa Hortaliças). 2000 CASTELO BRANCO, M.; GUIMARÃES, A.L. Controle da traça-das-crucíferas em repolho, 1989. Horticultura Brasileira, Brasília, v. 8, n. 1, p. 2425, 1990.

CHENG, E.Y. Insecticide resistance study in Plutella xylostella L. II. A general survey (198081). Journal of Agricultural Research of China, v. 30, p. 285-293, 1981.

FINNEY, D.J. Probit anaysis. $3^{\text {a }}$ edição. Cambridge University Press. Cambridge. 1971 $333 \mathrm{p}$.
FRANÇA, F.H., MEDEIROS, M.A. Impacto da combinação de inseticidas sobre a produção de repolho e parasitóides associados com a traça-dascruciferas. Horticultura Brasileira, Brasília, v. 16, p. 132-135, 1998.

HAMA, H.; SUZUKI, K.; TANAKA, H. Inheritance and stability of resistance to Bacillus thuringiensis formulations of the Diamondback Moth, Plutella xylostella (Linnaeus) (Lepidoptera: Yponomeutidae). Applied Entomology and Zoology, v. 27, p. 355-362, 1992.

IQBAL, M.; VERKERK, R.H.J.; FURLONG, M.J.; ONG, P.C.; RAHMAN, S.A.; WRIGHT, D.J. Evidence for resistance to B. thuringiensis (B.t.) subsp. kurstaki HD-1, B.t.subsp. aizawai and abamectin in field populations of Plutella xylostella from Malaysia. Pesticide Science, v. 48, p. 89-97, 1996.

LIU, M.Y.; TZENG, Y.J.; SUN, C.N. Diamondback Moth resistance to several synthetic pyrethroids. Journal of Economic Entomology, v. 74, p. 393-396, 1981

SHELTON, A.M.; WYMAN, J.A.; CUSHING, N.L.; APFELBECK, K.; DENNEHY, T.J.; MAHR, S.E.R.; EIGENBRODE, S.D. Resistance of Diamondback Moth (Lepidoptera: Plutellidae) to Bacillus thuringiensis subsp. in the field. Journal of Economic Entomology, v. 86, p. 697705, 1993.

SUDDERUDDIN, K.J.; KOK, P.F. 1978 Insecticide resistance in Plutella xylostella collected from the Cameron Highlands of Malaysia. FAO Plant Protection Bulletin, v. 26, p. 53-57, 1978.

TABASHNIK, B.E.; CUSHING, N.L.; JOHNSON, M.W. Diamondback Moth (Lepidoptera: Plutellidae) resistance to insecticides in Hawaii: intra-island variation and cross resistance. Journal of Economic Entomology, v. 80, p. 1091-1099, 1987.

ZHANG, X.Y.; JIE, H.; YE, C.Y.; XUE, Y.: Monitoring on resistance of diamond back moth to abamectin and field control experiments in Yunnan, China. Journal of Huazhong Agricultural University, v. 20, p. 426-430, 2001. 